\title{
openEHR-to-FHIR: Converting openEHR Compositions to Fast Healthcare Interoperability Resources (FHIR) for the German Corona Consensus Dataset (GECCO)
}

\author{
Nektarios LADAS ${ }^{\mathrm{a}, 1}$, Stefan FRANZ ${ }^{\mathrm{a}}$, Birger HAARBRANDT ${ }^{\mathrm{a}}$, \\ Kim Katrin SOMMER ${ }^{\mathrm{a}}$, Severin KOHLER ${ }^{\mathrm{b}}$, Sarah BALLOUT ${ }^{\mathrm{a}}$, Johanna FIEBECK ${ }^{\mathrm{c}}$, \\ Michael MARSCHOLLEK ${ }^{\mathrm{a}}$ and Matthias GIETZELT ${ }^{\mathrm{a}}$ \\ ${ }^{a}$ Peter L. Reichertz Institute for Medical Informatics, TU Braunschweig and Hannover \\ Medical School, Germany \\ b Digital Health Charite, Berlin, Germany \\ ${ }^{\mathrm{c}}$ Center for Information Management, Hannover Medical School, Germany
}

\begin{abstract}
The German Corona Consensus (GECCO) established a uniform dataset in FHIR format for exchanging and sharing interoperable COVID-19 patient specific data between health information systems (HIS) for universities. For sharing the COVID-19 information with other locations that use openEHR, the data are to be converted in FHIR format. In this paper, we introduce our solution through a web-tool named "openEHR-to-FHIR" that converts compositions from an openEHR repository and stores in their respective GECCO FHIR profiles. The tool provides a REST web service for ad hoc conversion of openEHR compositions to FHIR profiles.
\end{abstract}

Keywords. GECCO: German Corona Consensus Data Set, openEHR, FHIR, COVID-19, interoperability

\section{Introduction}

In Hannover Medical School, the GECCO dataset is stored in the openEHR format, which is modeled based on the GECCO FHIR profile [1, 2]. To exchange the data, it has to be converted to the FHIR R4 format.

In this paper, we introduce our solution through a tool named "openEHR-to-FHIR" for converting and sharing the COVID-19 patient information.

\footnotetext{
${ }^{1}$ Corresponding Author, Nektarios Ladas, Hannover Medical School, PLRI-OE 8420, Carl-NeubergStraße 1, 30625 Hannover, Germany; E-mail: ladas.nektarios@mh-hannover.de.
} 


\section{Methods}

The data model is based on two main compositions from the openEHR Clinical Knowledge Manager templates [3, 4].

Each GECCO composition is mapped with a Java class that contains the JSON code equivalent to an FHIR resource based on the GECCO - Implementation Guide [5].

Through the application of regular expressions, each element in FHIR is matched with the corresponding element in composition flat format [6] of the openEHR.

\section{Results}

From a total of 4 compositions, data for a total of 30 compositions are selected and separated. The GECCO openEHR compositions are then converted to 66 datasets to match the definition in FHIR R4 format and stored on HAPI FHIR server.

The converted dataset is shared through the HiGHmed Data Sharing Framework for exchanging COVID-19 specific information between university hospitals in Germany [7].

\section{Discussion}

Our approach provides a solution for converting compositions from an openEHR repository to FHIR format directly. The manual mapping for generating FHIR format datasets helps to easily modify and update changes on the data structure.

This approach can be used for converting any openEHR data to FHIR data. The source code for openEHR-to-FHIR is available at https:// gitlab.plri.de/NektariosLadas/openehr-to-fhir.

\section{References}

[1] Sass J, Bartschke A, Lehne M, Essenwanger A, Rinaldi E, Rudolph S, Heitmann KU, Vehreschild JJ, von Kalle C, Thun S. The German Corona Consensus Dataset (GECCO): a standardized dataset for COVID-19 research in university medicine and beyond. BMC Medical Informatics and Decision Making. 2020 Dec;20(1):1-7.

[2] German Corona Consensus Data Set - Implementation Guide [Internet]. Simplifier.net. 2021 [cited 20 August 2021]. Available from: https://simplifier.net/guide/GermanCoronaConsensusDataSetImplementationGuide/GECCOCore.

[3] Clinical Knowledge Manager 1 [Internet]. Ckm.highmed.org. 2021 [cited 19 August 2021]. Available from: https://ckm.highmed.org/ckm/templates/1246.169.1443

[4] Clinical Knowledge Manager 2 [Internet]. Ckm.highmed.org. 2021 [cited 19 August 2021]. Available from: https://ckm.highmed.org/ckm/templates/1246.169.1440

[5] GECCO - Implementation Guide [Internet]. Simplifier.net. 2021 [cited 25 August 2021]. Available from: https://simplifier.net/guide/germancoronaconsensusdataset-implementationguide/home

[6] Simplified Data Template (SDT) [Internet]. Specifications.openehr.org. 2021 [cited 25 August 2021]. Available from: https://specifications.openehr.org/releases/ITSREST/latest/simplified data template.html

[7] Hund H, Wettstein R, Heidt CM, Fegeler C. Executing Distributed Healthcare and Research ProcessesThe HiGHmed Data Sharing Framework. InGerman Medical Data Sciences: Bringing Data to Life 2021 (pp. 126-133). IOS Press. 